\title{
Enhancing the Performance of TCP by Improvising on the Key MANET Routing Protocols
}

\author{
Aniket Deshpande ${ }^{1}$ and Dr. Ashok Kaushal ${ }^{2}$
}

\begin{abstract}
Mobile Ad-hoc Networks has dynamic nature and multi-hop, in which each station frequently alters its position by configuring itself automatically. The constraint for exchange of info or data over the wireless settings is quickly increasing [1]. Currently, there is a growing need for accessing the Internet on various connections as well as the desire to improve TCP performance in Ad-hoc Networks; thus, there is a need for End-to-end Approaches Protocol; TCP DOOR (Out of Order Delivery Event Protocol); Feedback Scheme Protocol; and Adaptive Back off Response Approach Protocol for effective TCP performance improvement in Ad-Hoc Network, and reliable routing protocol to guarantee a consistent and effective end-to-end data packet transmission of information across MANET (Mobile Ad-hoc Network) networks and wireless networks. However, the main challenge in MANET is to select the most effective, reliable, accurate, and the most important protocol that will play a maximum role for TCP performance improvement in an Ad-Hoc Network than in any other protocols. Existing literature or research studies have primarily focused on the simulation-based assessment of variants of TCP and those of TCP routing protocols' performance in MANETS as well as the use of other routing protocols that are required for improving TCP performances in Ad-Hoc Networks. This research analyses the four important routing protocols as identified by various researchers in the past for further analyzing and improvising on them with an objective of improving performances of TCP in Ad-Hoc Networks when compared against the current options [2]. These include: End-to-end Approaches Protocol; TCP DOOR (Out of Order Delivery Event Protocol); Feedback Scheme Protocol; and Adaptive Back off Response Approach Protocol. Based on the analysis, this study identifies whether creating a new protocol / algorithm combining the Feedback Scheme Protocol and Adaptive Back-Off is better than any of the 4 individual protocols and if so, the optimal way to do so.
\end{abstract}

Keywords - Ad-hoc Networks, TCP performance, End-to-end Approaches Protocol, TCP DOOR (Out of Order Delivery Event Protocol), Feedback Scheme Protocol, Adaptive Back off Response Approach Protocol

\section{INTRODUCTION}

MANET refers to a definite variety of wireless network that involves the group of mobile nodes that have the ability to communicate with one other with no assistance deriving from secure/fixed infrastructures [3]. Transmission Control Protocol is designed for Ad-Hoc as well as other wired networks. However, due to the emerging technologies like IOT, Cloud-based services, etc. that are primarily wireless Ad-Hoc connectivity driven, the requirement to adopt Transmission Control Protocol in Ad-Hoc Networks has gained significance. But this pops up a few challenges as the Transmission Control Protocol has poor performance in MANET because of dynamic topology, shared medium, multi-hop architecture, high-error ratio, and channel connotation [4]. Therefore, for Adhoc networks to efficiently operate, appropriate protocol level controls should be incorporated to bring in efficiencies in the performance of TCP in Ad-Hoc Networks. Additionally, this research also seeks to justify why the 4 protocols, namely, End-to-end Approach Protocol, TCP DOOR (Out of Order Delivery Event Protocol), Feedback Scheme Protocol and the Adaptive Back-off Response Approach Protocol [2] are the more critical relative

\footnotetext{
${ }^{1}$ Research Scholar, Department of Computer Science \& Engineering, Faculty of Engineering and Technology Mewar University, NH-79, Gangrar, Chittorgarh, Rajasthan, India

${ }^{2}$ Research Scholar, Department of Computer Science \& Engineering, Faculty of Engineering and Technology Mewar University, NH-79, Gangrar, Chittorgarh, Rajasthan, India
} 
to any of the other protocols for enhancing the TCP performance in MANETs. This secondary research based justification will focus on evaluating the importance of these protocols in improving Transmission Control Protocol performances in Ad-Hoc Networks is also included in this research. This will also help in identifying if any of the 4 individual protocols discussed in this paper or their hybrid derivative will further bring enhancement to the TCP performance compared to the current levels.

\section{Protocols USED to improve PeRformance OF TCP IN MObILE AD-Hoc NetWorkS}

\section{A. TCP-DOOR (Out of Order Delivery Event Protocol) -}

TCP-DOOR (Out of Order Delivery Event Protocol) [5] attempts to improve the performances of TCP in ad hoc networks by identifying and giving proper response to the occurrences of out of order packet delivery; therefore, TCP-DOOR avoids invoking the congestion control mechanism that is not necessary by definition, OOO (out-oforder) usually take place when a sent pack or package first reaches the destination after the subsequent packet, which was sent later. In Ad-Hoc Networks, out-of-order could occur several times in a single TCP session due to changes of the route. Therefore, to effectively detect out-of-order delivered packets, an ordering info is added to TCP datapackets and TCP ACKs.

The detection of OOO is conducted at the two sides; where the receiver identifies the OOO data and packs or packages whereas the OOO ACK packs are detected in the end of the sender. The receiver will then notify the sender if it detects an out-of-order receipt of a packet, since the sender is responsible for invoking congestion control mechanism. If the sender identifies any out-of-order conditions, TCP DOOR might respond quickly to temporarily deactivate congestion control as well as provide an immediate retrieval action during the evasion or prevention of congestion.

General issues: The key reason for using TCP DOOR is to detect of OOO (out-of- order) condition and highlight that the route change event has just happened. On the other hand, out-of-order could only be detected when the routes have recuperated from errors or a failure. Consequently, TCP-DOOR is not responsive and accurate as the feedbackbased approach protocol that has the ability to determine whether route errors or congestion has occurred, and therefore account/report to the sender(s) precisely at the very start. In addition, TCP DOOR might not perform well with various multi-path routings because a multi-path routing might as well cause OOO. Hence, it is suggested that TCP-DOOR can be used to substitute or replace the feedback scheme protocol [5] for enhancing TCP performance in MANETs, only when Feedback Scheme Protocol or feedback-based approach is not available.

\section{B. Feedback Scheme Protocol}

Invoking congestion control mechanism to treat the route failure scenario as equivalence to the occurrence of congestion is not recommended as the route failure and congestion control are totally different situations that need to be addressed differently. The use of Feedback Scheme Protocol through which the route failure is reported to the source will not cause unnecessary congestion control. Feedback Scheme Protocol also halts distribution of other or more packets till the routes are completely reinstated. The use of Feedback based schemes for improving the performance of TCP have been initially recommended in the form of EBSN (European Basic Skills Network) [6] in cellular networks and ECN (explicit congestion notification) [7] for fixed networks. However, there is no reliable literature study for the use of Feedback based schemes to enhance the performance of TCP over the Mobile Ad-Hoc Network. Hence, this research discusses the Feedback based scheme Protocol to improve performances of TCP in AdHoc Networks, and deal with the failures of routes in Ad-hoc Networks known as Transmission Control Protocol Feedback)

General issues: Due to unpredictable and frequent route failures, the performance of TCP over MANETs is considerably reduced both in terms of good put (which is the ratio of the whole data transmitted by the source and the valuable information/data received at the end) and throughput [6]. Therefore, a feedback scheme protocol can be used to overcoming the route failure issues and increase the performance of TCP. Thus, using feedback scheme protocol may lead to significantly help bring improvement in the transmission layer of MANET.

\section{End-to-end Approaches Protocol}

TCP's End-to-end Approach Protocol offers byte stream transport mechanisms, consistency, end-to-end congestion's control mechanisms, as well as the flow and congestion controls [8]. It improves medium contentions, particularly when the size of the congestion window surpasses the maximum values. 


\section{Adaptive Back-off Response Approach Protocol}

The Adaptive Back-off Response Approach protocol is also one of the approaches to enhance TCP performance in MANETs. The Adaptive Back-off response approach protocol adds an extra MAC-layer delay(s) equivalent to the time of transmission of a single pack or package that alleviates inter-packet interference in Mobile Ad-hoc Networks.

The Adaptive Back-off response approach protocol is also a reliable multicast transport protocol that is designed to acclimatize various conditions of Mobile Ad-hoc Networks; this allows Adaptive- back off response to enhance TCP's performance in these networks (MANETs). Adaptive- back off response approach protocol implements a source-based recovery scheme through the use of error and congestion control to prevent and recover from packet losses as a result of congestion. Moreover, adaptive- back off response approach protocol can perform local recovery at inter-mediate nodes to recover from losses not essentially as a result of congestion.

In the event of a retransmission time-out (RTO), TCP re-transmits the earliest packet that wasn't acknowledged and subsequently increases the RTO's intervals by two times. This procedure is replicated till ACKs have been received for the packet that was retransmitted. Therefore, RTO interval could likely be longer even if the routes may have been redeveloped or created earlier. This situation results in the wastage of time that could have been better used for sending a packet (or pack). In order to improve TCP's performance, this study attempts to minimize the time thus wasted, by ensuring that all RTO intervals rely on SRTT, which is the average mean of an evaluated RTO. The Adaptive Back-off response approach protocol [9] usually relies on conserving the values of the congestion window, smoothed round trip time and the slow start threshold, especially when the retransmission timer elapses. Hence instead of doubling the RTO interval every time, the study multiplies it by a value between One and Two known as

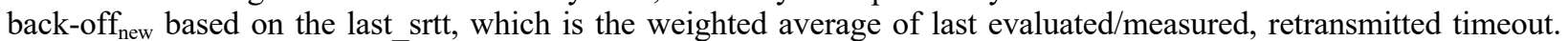
The resulting Back-off new $_{\text {and }} \mathrm{RTO}_{\text {new }}$ value (the new RTO) were calculated as follows:

$$
\begin{gathered}
\text { Back off new }=1+\frac{\text { (last_sstt }- \text { min_srtt) }}{\text { (max_sstt } \left.-m i n_{-} s r t t\right)} \\
R T O_{\text {new }}=\text { Backoff } f_{\text {new }} * R T O_{\text {current }}
\end{gathered}
$$

Whereby: $\mathrm{RTO}_{\text {current }}$ refers to the existing value of RTO; while min._srtt (Minimum smoothed round trip time) refers to the least SRTT that is observed; and max._srtt (Maximum smoothed round trip time) is the value observed at the peak. The study initialized max_srtt and min_s rtt with the $0.6 \mathrm{sec}$ and $0.1 \mathrm{sec}$ values respectively, selecting the values for initializing the two variables [10].

\section{EXPERIMENTAL OUTCOME OF THE PROPOSED COMBINATION OF FEEDBACK SCHEME AND ADAPTIVE BACK- off Response APPROACH PRotocol}

In Transmission Control Protocol New Reno, this study did simulation for Adaptive- back off response approach protocol dependent on the pause time, the number of node(s), simulation period, network areas, routing protocols, and the node speed. The investigation methodology comprised of difference in some of the key parameters like the pause time and node's speed. The number of node(s) were calculated periodically along with their impact on the performance of diverse TCP variations that were then evaluated or measured while keeping constant various factors such as the network area(s), simulation period, the pause time, node speed(s) and routing protocols. The evaluation of the simulation effects of Adaptive Back-off response approach protocol on TCP-New Reno's performance in MANETs as well as on other performances of TCP's protocols under a given experimented setting are illustrated graphically as shown below:

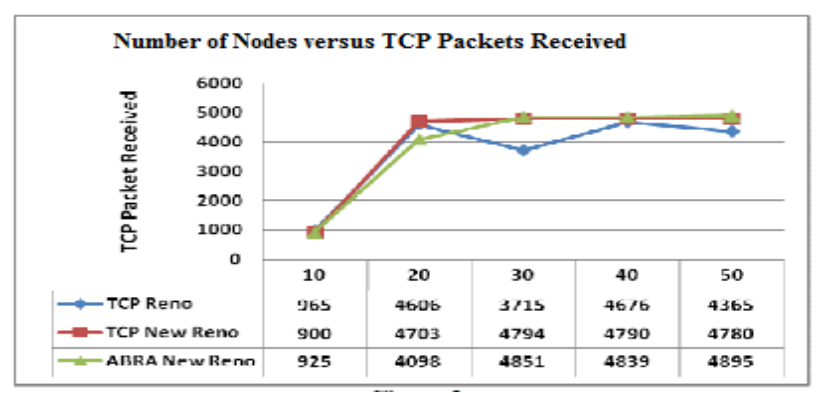

Figure 1. No. of Nodes Vs. No. of Packets Received 


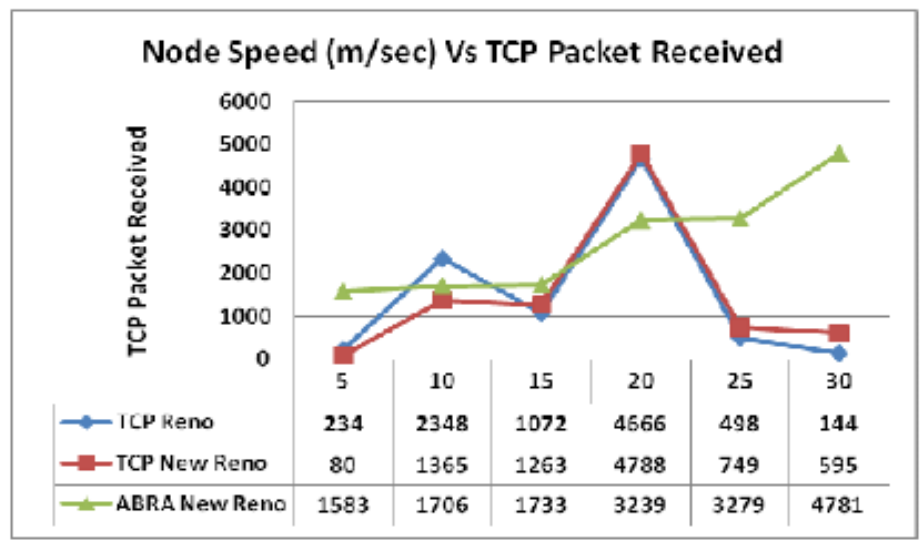

Figure 2. Node Speed Vs. No. of Packets Received

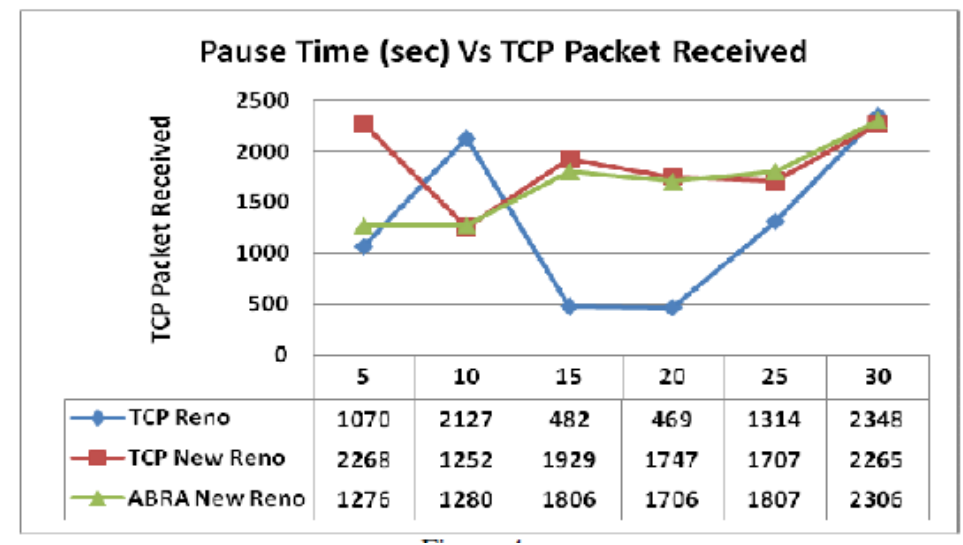

Figure 3. Pause Time Vs. No. of Packets Received

From the simulation outcomes shown in figures 1-3, it is seen that under the least number of nodes, pause time of the sender and the speed of node does not determine the appropriate path as the receiver(s) was distant from the sender (or source). Therefore, time-out cropped up in the numbers of mobile nodes in between receiver and sender resulting in least packets being received at the end. However, the increase in the variant factors e.g. increasing the number of nodes and node's speed as well as the network's pause time will result in multi-path options for the sender between the receiver and sender minimizing issues and thus, maximum packets will be received by the receiver.

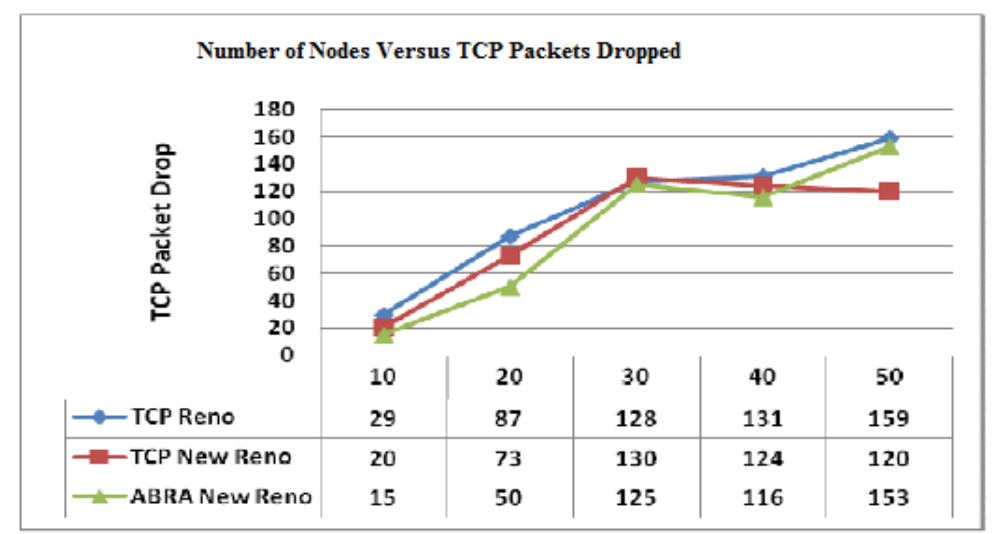

Figure 4. Number of Vs. No. of Packets Dropped 


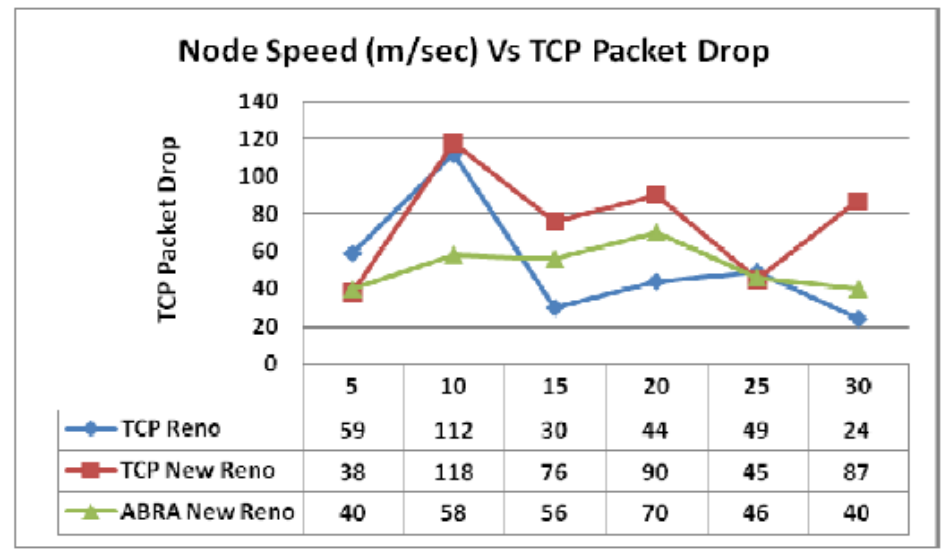

Figure 5. Node Speed Vs. No. of Packets Dropped

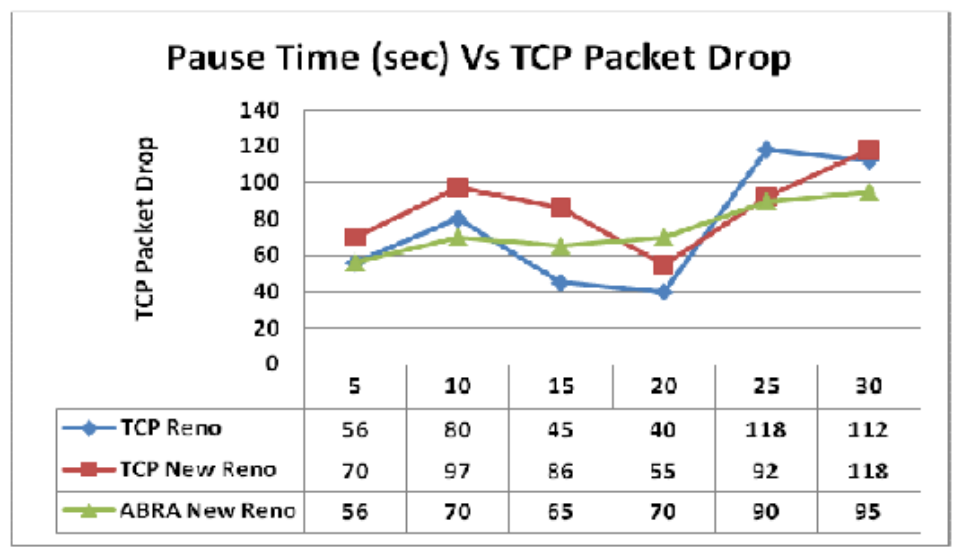

Figure 6. Pause Time Vs. No. of Packets Dropped

Simulation outcomes in the above figures (4-6) demonstrate that originally the packet drops are less due to less congestion, low route failure, less timeout waiting time, etc. However, with the increase of variants such as the number of nodes, speed of node as well as the network's pause time, the rate of packet drops also increase progressively as a result of high congestion, route failures, lack to reach the desired endpoint i.e. inaccessible destinations, expiration of provided time, frequently altered bandwidth or route change, node mobility, inappropriate routing path, etc.

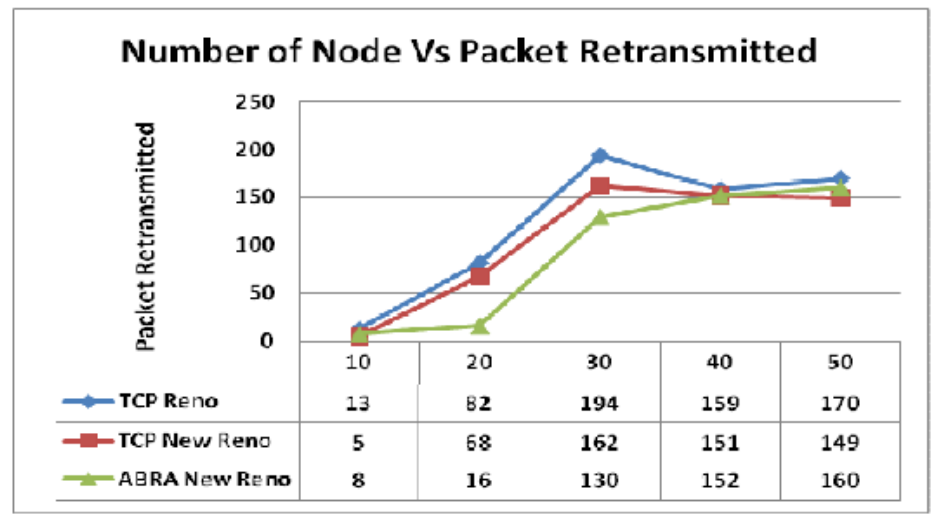

Figure 7. Number of Nodes Vs. No. of Packets Retransmitted 


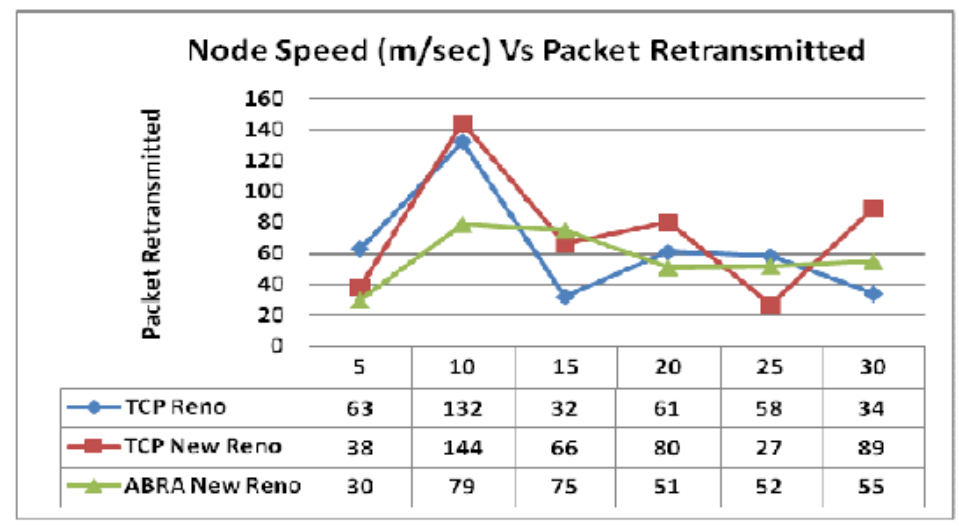

Figure 8. Node Speed Vs. No. of Packets Retransmitted

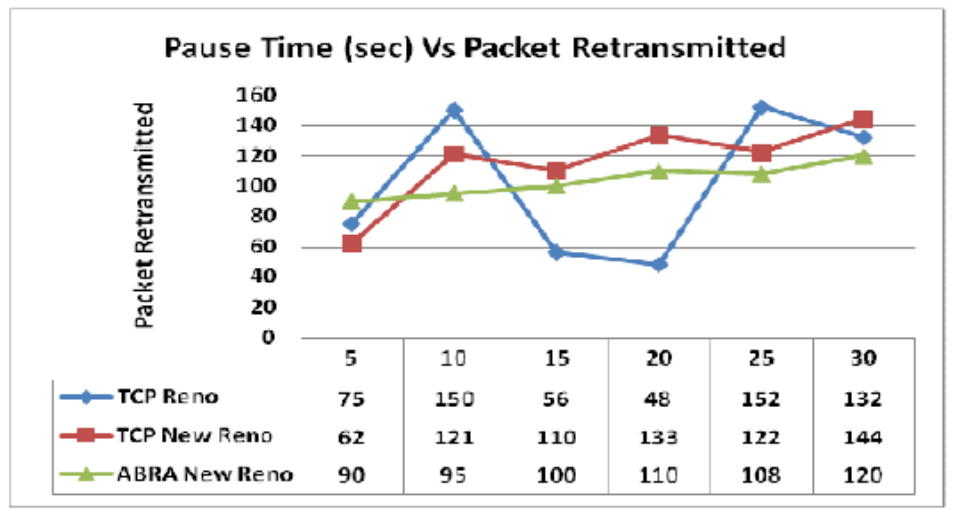

Figure 9. Pause Time Vs. No. of Packets Retransmitted

Simulation outcomes in figure7-9 illustrate the packets retransmitted upon dissimilar variants such as the variation in pause time, in network node speed, and the number of nodes. When the number of nodes is increased from 10 to 40 , packet retransmission is also increased progressively. However, the rate of packet retransmission varies under the increased pause time and the node mobility as a direct result of the increased rate of packet drops, packet delay, and the loss of acknowledgment as well as by time-out conditions.

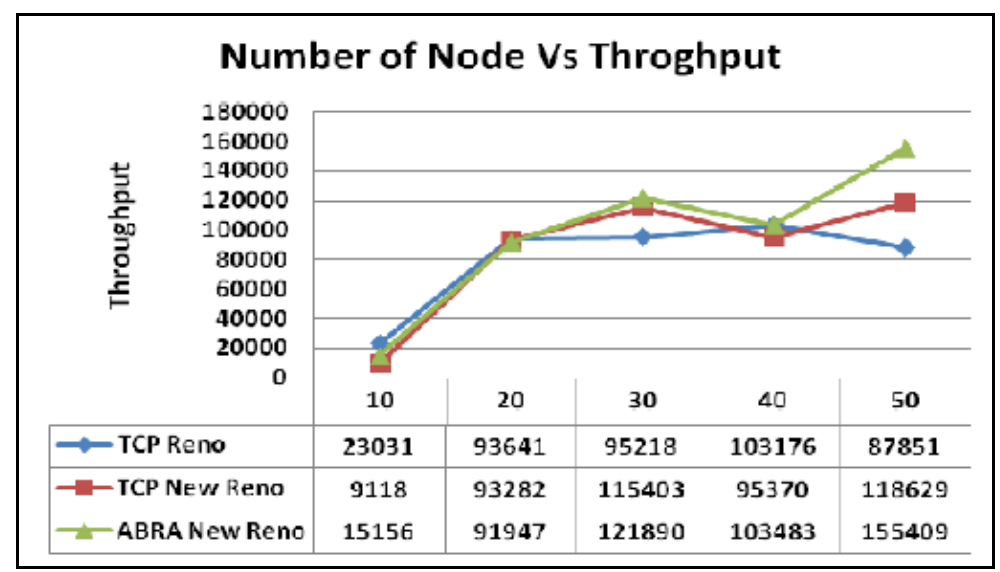

Figure 10. No. of Nodes Vs. Throughput 


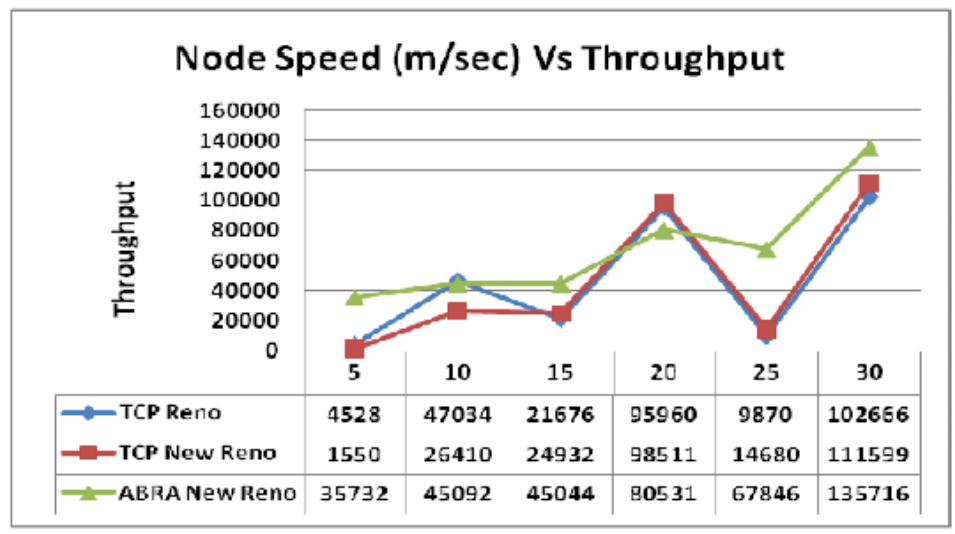

Figure 11. No. of Nodes Vs. Throughput

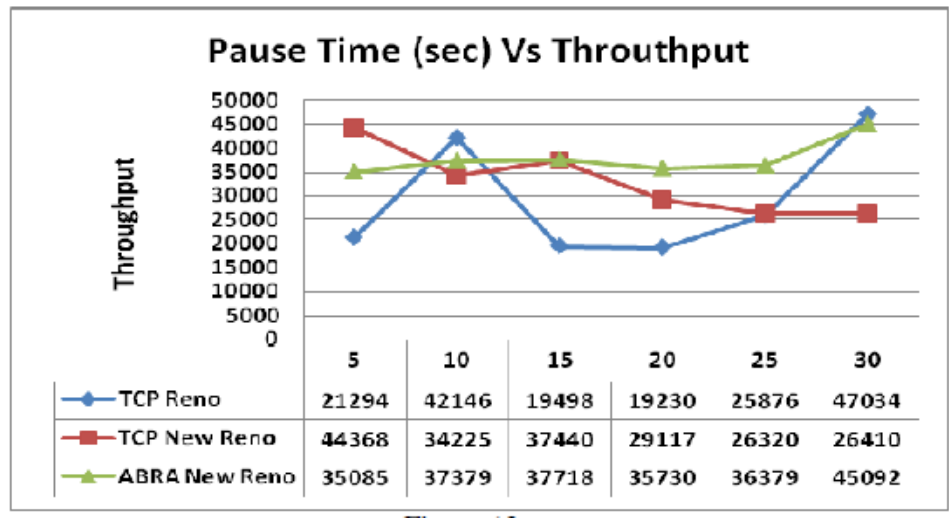

Figure 12. No. of Nodes Vs. Throughput

Simulation outcomes in figure 10-12 demonstrate the throughput of diverse variants with the difference in the pause time, node's speed as well as the number of nodes in Ad-hoc networks. Therefore, this shows that the effects of Adaptive Back-off response approach protocol on TCP performance (TCP New Reno) varies depending on the variations in the pause time, the number of nodes as well as node's speed. Moreover, the impact of Adaptive Back-off response approach protocol on TCP performance (TCP New Reno) varies according to the drop in acknowledgements, bytes received and the packets lost. Conversely, variants can be greater and sometimes inconsistent due to the delay (or latency) and the incorrect estimation of the bandwidth

\section{CONCLUSION}

Therefore, the new protocol that combines the Feedback and the Adaptive Back-off scheme can be used to improve the TCP performances over Ad-hoc networks by spotting or diagnosing as well as respond to the Out-of-Order instances of packet deliveries that are likely to occur due to the regular changes of routes. The study findings show that the Adaptive Back-off response approach protocol approach attained nearly 50\% improvement in TCP performance at the transport layer without needing any changes in the lower layers of the OSI model.

Moreover, the new protocol that combines the Feedback and Adaptive scheme could be used to address the problem of needing sufficient identical or additional acknowledgements to effectively identify any packet losses. Similarly, it can also address the issue of TCP slow start after back-off that may help to prevent it from congesting the entire network [9]. Thus this is definitely expected to help improve the TCP performance in Mobile Ad-hoc networks.

\section{Scope For Future Work}

This study recommends that in forthcoming efforts, the simulation of Adaptive Back-off response approach protocol when deployed together with Feedback scheme for additional parameters in realistic and larger scenarios will have a 
greater potential. Additionally, further simulations need to be performed in future to examine the effects of Adaptiveback off response approach protocol Transmission Control Protocol-New Reno on the other variants of TCP that may have a direct impact on parameters like latency, bandwidth and congestion window. Future work should focus on experimenting with various flows of Transmission Control Protocols in order to correctly or accurately simulate settings of crowded or jammed networks.

\section{REFERENCES}

[1] M. A. Rahman, F. Anwar, J. Naeem, and M. S. M. Abedin, "A simulation based performance comparison of routing protocol on Mobile Ad-hoc Network (proactive, reactive and hybrid)," in International Conference on Computer and Communication Engineering (ICCCE'10), pp. 1-5, 2010.

[2] A. Deshpande and A. Kaushal, "Key Performance Indicators of major TCP Routing Protocols Deployed in MANETs Aniket,” Int. J. Appl. or Innov. Eng. Manag., vol. 5, no. 4, pp. 54-58, 2016.

[3] J. Liu and S. Singh, “ATCP: TCP for mobile ad hoc networks,” IEEE J. Sel. Areas Commun., vol. 19, no. 7, pp. 1300-1315, Jul. 2001.

[4] K.-C. Leung and V. Li, "Transmission control protocol (TCP) in wireless networks: issues, approaches, and challenges,” IEEE Commun. Surv. Tutorials, vol. 8, no. 4, pp. 64-79, 2006.

[5] F. Wang and Y. Zhang, "Improving TCP performance over mobile ad-hoc networks with out-of-order detection and response," in Proceedings of the 3rd ACM international symposium on Mobile ad hoc networking \& computing - MobiHoc '02, p. 217, 2002.

[6] B. Bakshi, P. Krishna, N. H. Vaidya, and D. K. Pradhan, "Improving Performance of TCP over Wireless Networks," in Proceedings of the 17th International Conference on Distributed Computing Systems (ICDCS '97),, p. 596, 1997

[7] S. Floyd and Sally, "TCP and explicit congestion notification," ACM SIGCOMM Comput. Commun. Rev., vol. 24, no. 5, pp. 8-23, Oct. 1994.

[8] A. Al Hanbali, E. Altman, and P. Nain, "A survey of TCP over ad hoc networks," IEEE Commun. Surv. Tutorials, vol. 7, no. 3, pp. 22-36, 2005.

[9] M. N. I. Khan and R. Ahmed, Simulation Based Performance Evaluation in MANET: Routing Protocols and TCP variants, 2011th ed. VDM Verlag, 2011.

[10] M. N. I. Khan, R. Ahmed, and M. T. Aziz, "A SURVEY OF TCP RENO, NEW RENO AND SACK OVER MOBILE AD-HOC NETWORK,” Int. J. Distrib. Parallel Syst., vol. 3, no. 1, pp. 49-63, 2012. 